\title{
Rapid Whole Genome Sequencing of Serotype K1 Hypervirulent Klebsiella pneumoniae from an Undocumented Chinese Migrant
}

\author{
C. K. Macleod, ${ }^{1,2}$ F. A. Khokhar, ${ }^{3}$ B. Warne ${ }^{3}$ R. Wick $\left(\mathbb{D},{ }^{4}\right.$ R. Butcher $\mathbb{D}^{1},{ }^{1}$ B. Cassimon, ${ }^{2}$ \\ P. Hayden, ${ }^{2}$ K. Holt, ${ }^{4,5}$ and M. E. Török (DD ${ }^{3,6}$ \\ ${ }^{1}$ Department of Clinical Research, London School of Hygiene \& Tropical Medicine, London, UK \\ ${ }^{2}$ Medway Maritime Hospital, Gillingham, Kent, UK \\ ${ }^{3}$ Department of Medicine, University of Cambridge, Cambridge, UK \\ ${ }^{4}$ Department of Infectious Diseases, Central Clinical School, Monash University, Melbourne, Australia \\ ${ }^{5}$ Department of Infection Biology, London School of Hygiene \& Tropical Medicine, London, UK \\ ${ }^{6}$ Public Health England, Clinical Microbiology and Public Health Laboratory, Cambridge, UK \\ Correspondence should be addressed to M. E. Török; estee.torok@gmail.com
}

Received 22 November 2020; Revised 11 February 2021; Accepted 30 March 2021; Published 28 April 2021

Academic Editor: Saikat Majumder

Copyright ( 92021 C. K. Macleod et al. This is an open access article distributed under the Creative Commons Attribution License, which permits unrestricted use, distribution, and reproduction in any medium, provided the original work is properly cited.

Background. Hypervirulent Klebsiella pneumoniae causes severe disseminated infections, typically with hepatic and central nervous system involvement including endophthalmitis. Case Presentation. We report a fatal case of an undocumented Chinese migrant in her 60s who presented to the emergency department with abdominal pain, lethargy, and headache over the preceding two weeks. She had a new diagnosis of diabetes mellitus on admission. Computed tomography scan of the thorax, abdomen, and pelvis showed bilateral pneumonia with liver abscesses. The patient was treated with empirical broad-spectrum antibiotics before K. pneumoniae was isolated from cerebrospinal fluid and blood cultures. The isolate was further characterised as a ST23 (ST: sequence type), serotype K1 hypervirulent strain using Nanopore sequencing. Despite admission to the intensive care unit, the patient died within 48 hrs of admission. Conclusions. This case demonstrates the need for increased awareness of hypervirulent K. pneumoniae, even in countries where it occurs infrequently. Novel, rapid, sequencing technologies can support diagnosis in unusual presentations.

\section{Background}

Classical Klebsiella pneumoniae (cKp) is an opportunistic pathogen that typically causes urinary tract infections and is a leading cause of nosocomial pneumonia. cKp infections are most common amongst those at the extremes of age or in those who are immunosuppressed. In contrast, hypervirulent $K$. pneumoniae (hvKp) causes severe disseminated infections in otherwise healthy individuals [1]. hvKp is associated with pyogenic liver abscesses as well as pneumonia, meningitis, and endophthalmitis. The strain is rarely seen in Northern Europe, with only occasional case reports in the literature; however, colonisation studies have found hvKp in $0.0-16.7 \%$ of community members in South-East Asian countries [2-6]. Although hvKp has been associated with increased viscosity of colonies on agar plate, there is no reliable way to distinguish hvKp from cKp phenotypically and so a high degree of clinical suspicion is required for rapid diagnosis and optimised treatment.

The decreasing cost and size of portable sequencing devices are of increasing interest in atypical clinical presentations of infectious diseases to rapidly genotype pathogens. Having this information available routinely in clinical microbiology laboratories could aid diagnosis and ensure rapid treatment.

We report the case of a woman with hepatic abscesses, bilateral pneumonia, and meningitis with $K$. pneumoniae grown on blood and cerebrospinal fluid culture.

The infection was unfortunately fatal, and subsequent sequencing using a portable nanopore-based device further characterised the isolate as a hypervirulent strain. 


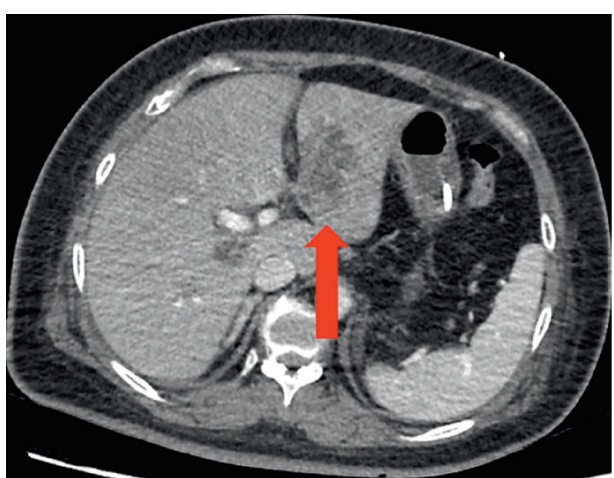

(a)

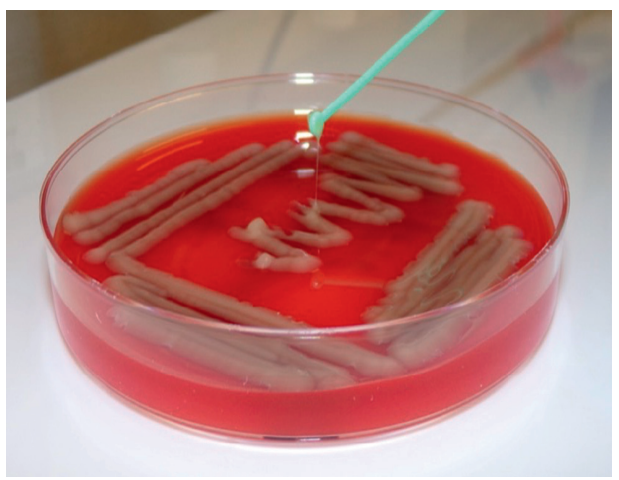

(c)

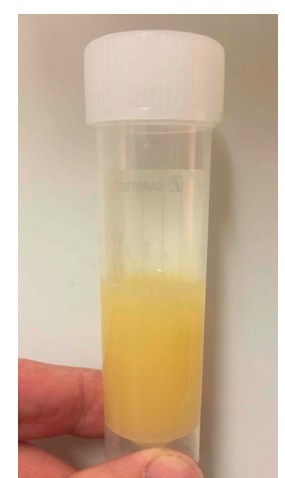

(b)

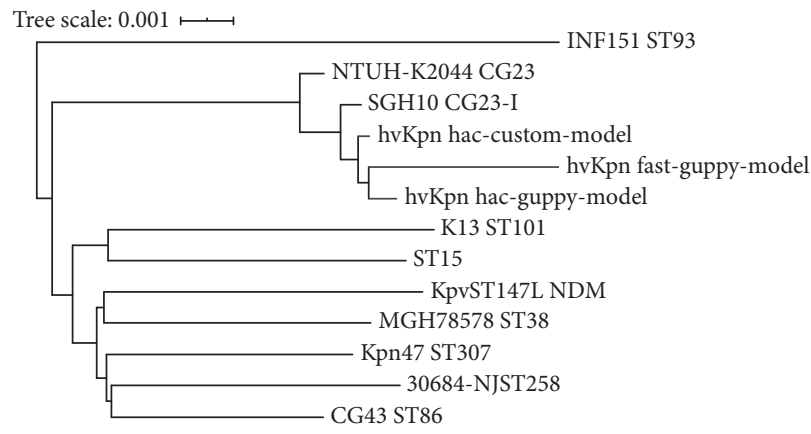

(d)

Figure 1: (a) Computed tomography scan of the abdomen showing multiple liver abscesses. (b) Universal container with turbid cerebrospinal fluid. (c) Blood agar plate with Klebsiella pneumoniae culture. (d) Genome distance tree comparing the clinical hypervirulent $K$. pneumoniae isolate with 10 reference K. pneumoniae isolates produced using Mash.

\section{Case Presentation}

A female patient in her 60 s presented to a hospital emergency department with a 24-hour history of vomiting. She had a two-week history of right upper quadrant abdominal pain and severe frontal headache. She did not report diarrhoea or weight loss and had no history of neck stiffness, photophobia, rash, or limb weakness. She had been generally well since arriving in the United Kingdom (UK) from China 18 months ago. She had no relevant medical history of chronic disease and she was not formally employed. No other family members had been unwell recently, and she reported no foreign travel since arriving in the UK.

Blood tests on admission showed a mild leucocytosis, with a white blood cell (WBC) count of $10.6 \times 10^{9} / \mathrm{L}$ (neutrophils $9.5 \times 10^{9} / \mathrm{L}$, lymphocytes $0.7 \times 10^{9} / \mathrm{L}$, and eosinophils $0.1 \times 10^{9} /$ $\mathrm{L}$ ), and severe thrombocytopaenia (platelets $17 \times 10^{9} / \mathrm{L}$ ). Liver function tests were deranged with increased bilirubin $21 \mu \mathrm{mol} /$ $\mathrm{L}$, alanine aminotransferase $169 \mathrm{IU} / \mathrm{L}$, and alkaline phosphatase $337 \mathrm{IU} / \mathrm{L}$. Coagulation was abnormal with a prothrombin time of 10.4 seconds and a fibrinogen level $>9.3 \mathrm{~g} / \mathrm{L}$. Other biochemical tests were unremarkable, with normal sodium $(140 \mathrm{mmol} / \mathrm{L})$, potassium $(3.1 \mathrm{mmol} / \mathrm{L})$, and creatinine $(63 \mu \mathrm{mol} / \mathrm{L})$ and a mildly raised urea $(9.3 \mathrm{mmol} / \mathrm{L})$. The C-reactive protein concentration was very high at $250.6 \mathrm{mg} / \mathrm{L}$. A urine dipstick was negative for leucocytes, nitrites, and blood, but showed high $(2+)$ glucose and very high (3+) ketones.
The patient was treated as presumed biliary sepsis with intravenous amoxicillin and clavulanic acid (co-amoxiclav; $1.2 \mathrm{~g}$ intravenous three times daily). She was admitted to the acute medical unit and blood was sent for microbial culture. An ultrasound of the abdomen showed multiple solid, mixed echogenicity lesions in both lobes of the liver, the largest of which measured $41 \times 34 \mathrm{~mm}$, a normal gallbladder, and nondilated common bile duct. A computed tomography (CT) scan of the brain was reported as normal.

Twelve hours after admission, the patient became less responsive with a Glasgow Coma Scale 7/15 (E2 V2 M3). Venous blood gas showed metabolic acidosis ( $\mathrm{pH} 7.12 ; \mathrm{HCO}_{3}$ $8 \mathrm{mmol} / \mathrm{L}$ ), hyperglycaemia (glucose $29.1 \mathrm{mmol} / \mathrm{L}$ ), and marked ketosis (ketones $7.0 \mathrm{mmol} / \mathrm{L}$ ). She was intubated for airway protection and started on an intravenous insulin and fluid resuscitation regime for diabetic ketoacidosis. She was given empirical treatment for presumed central nervous system infection with cefotaxime $2 \mathrm{~g}$ IV four times daily and aciclovir $10 \mathrm{mg} / \mathrm{kg}$ IV three times daily. A repeat CT scan of the brain was reported as normal, and she was admitted to the intensive care unit (ICU). A CT scan of the chest, abdomen, and pelvis showed bilateral pneumonia, appearances suggestive of an abscess within the left lobe of the liver, and moderate intraabdominal free fluid (Figure 1(a)). A blood film showed marked thrombocytopaenia with no fragments or clots. One pool of platelets was transfused in order to enable a lumbar puncture to be performed to collect cerebrospinal fluid (CSF). 
The CSF was turbid and yellow (Figure 1(b)), with a very high opening pressure of $>57$ centimetres of CSF (above the maximum recordable level of the manometer). Both the CSF protein and WBC count were markedly elevated at $11.8 \mathrm{~g} / \mathrm{L}$ and $50,000 / \mathrm{m}^{3}$ (85\% polymorphs and $15 \%$ lymphocytes), respectively. The CSF glucose was low at $1.0 \mathrm{mmol} / \mathrm{L}$, and the red blood cell count was $640 \mathrm{cells} / \mathrm{mm}^{3}$. The sample was positive for xanthochromia, but no organisms were seen on Gram stain or acid-fast stain. Blood and CSF cultures subsequently grew $K$. pneumoniae, which was reported as sensitive to cefotaxime and gentamicin. The CSF was PCR negative for $M$. tuberculosis, herpes simplex DNA, Zika virus RNA, and enterovirus RNA. A blood HIV test was negative. The HbAlc level was $100 \mathrm{mmol} / \mathrm{mol}$, suggestive of persistently high blood glucose levels in the preceding months, consistent with a new diagnosis of diabetes mellitus. Following admission to the ICU, the patient was found to have fixed and dilated pupils and, following brainstem testing, a decision was made to withdraw care. The patient died 18 hours later.

The blood culture isolate was cultured onto Columbia Blood Agar (Oxoid, UK) and incubated overnight at $37^{\circ} \mathrm{C}$. A single colony was picked and subcultured overnight. The antimicrobial susceptibility of the isolate was determined using the $\mathrm{N} 350$ card on the VITEK2 ${ }^{\circledR}$ instrument (bioMeriéux, France). DNA was extracted and sequenced on a MinION (Oxford Nanopore Technologies); details are shown in Supplemental Material.

Basecalling was performed using Guppy v3.4.4 with three approaches: a fast option with default settings, a high-accuracy option with a methylation-aware model, and a highaccuracy option with a custom model trained on Klebsiella genomes. All read sets were trimmed, filtered, and assembled using Flye v2.7 [7], and draft assemblies were further polished with Racon v1.3.1 [8] and Medaka v 0.7.1 to generate final consensus assemblies (available in FigShare, doi: 10.6084/ m9.figshare.11770908; see details in Supplemental Material).

Species confirmation and Klebsiella-specific genotyping including multilocus sequence typing (MLST), AMR, and virulence determinants were performed using Pathogenwatch (https://pathogen.watch), Kaptive (https://github. com/katholt/Kaptive), and Kleborate (https://github.com/ katholt/Kleborate) [9]. Reference genomes of common hypervirulent or antimicrobial-resistant $\mathrm{K}$. pneumoniae (Supplementary Table S1) were downloaded from NCBI and compared to the consensus assemblies by estimating pairwise genome distances using Mash [10] and then constructing a neighbour-joining tree in $\mathrm{R}$.

The blood culture isolate was hypermucoid (Figure 1(c)) and identified by matrix-assisted laser desorption/ionization time-of-flight mass spectrometry (MALDI-TOF MS) as $K$. pneumoniae. It was resistant to ampicillin but was susceptible to all other antimicrobials tested. Sequencing via MinION yielded (after basecalling and demultiplexing) $\sim 2$ million high-quality reads totalling $\sim 6 \mathrm{Gbp}$, giving $\sim 1150$ fold coverage of the genome. Average read length was $3.2 \mathrm{Kbp}$, maximum read length $70.6 \mathrm{Kbp}$, and N50 6.0 Kbp. All three assemblies resolved two circular molecules: a $5.4 \mathrm{Mbp}$ chromosome and a $228 \mathrm{Kbp}$ plasmid.
The genome sequence confirmed the species identification as $K$. pneumoniae. Distance-based phylogenetic analysis showed the novel isolate clustered with the ST23 (sequence type) K1 hypervirulent clinical isolates SGH10 [11] and NTUH-K2044 [12] and was closest to SGH10 which is representative of the dominant globally distributed liver abscessassociated subclade CG23-I (Figure 1(d)). Genotyping analysis of nanopore-only data is complicated by basecalling error rates $>0.1 \%$ [13]. Nevertheless, the genome sequence assembled from reads basecalled with a K. pneumoniae-trained custom model was identified as a single-locus variant of ST23, with virulence-associated features typical of CG23-I [14]: capsular serotype $\mathrm{K} 1$, the genotoxin colibactin (clb/pks), acquired siderophore systems yersiniabactin (ybt), aerobactin (iuc1), and salmochelin (iro1), plus rmpA and rmpA2 (associated with hypermucoidy). The assembly derived from default basecalling identified the presence of the loci but yielded less precise genotypes (see Supplementary Table S2). The iuc1, iro1, rmpA, and rmpA2 were located in the 228 plasmid, which showed full-length homology with pSGH10, a type I K. pneumoniae virulence plasmid (KpVP-1) typical of ST23 [9].

\section{Discussion and Conclusions}

We present the case of a patient with disseminated hvKp infection. hvKp causes disseminated disease and needs to be rapidly identified in clinical cases to prevent the devastating consequences of overwhelming infection. Rapid sequencing available via nanopore technology such as the MinION device enables prompt identification of pathogenic strains and allows clinicians to appropriately investigate and treat patients. The patient in this case had risk factors for hvKp, including Chinese origin, but had been residing in the UK for 18 months and had been apparently well prior to presentation. Infections caused by hvKp strains were initially reported from South-East Asia in the 1980s but have spread worldwide [15]. Diabetes mellitus is another well-recognised risk factor for K. pneumoniae liver abscess [16]; although the patient was not known to be diabetic prior to admission, she presented in diabetic ketoacidosis, with evidence of longterm poor glycaemic control.

We used a rapid sequencing platform to analyse the bacterial isolate and identified it as K. pneumoniae of capsular serotype K1 and sequence type ST23, which accounts for the majority of $K$. pneumoniae liver abscess cases and is widespread in South-East Asia. ST23 strains with the K1 capsule type are especially prevalent in China [17] and are those most commonly associated with progression to central nervous system infection [18]. The genome sequence was closely related to two previously reported ST23 liver abscess isolates, SGH10 (from a case in Singapore) [11] and NTUH-K2044 (from a patient in Taiwan with liver abscesses and meningitis) [12]. The isolate carried all the acquired virulence factors typical of ST23, including the virulence plasmid and chromosomally encoded colibactin (a marker of the major pyogenic liver abscess-associated sublineage, CG23-I) [11]. 
This work shows how the portable MinION device can be used to rapidly clarify the aetiology of unusual case presentations, in this case confirming that the infection was caused by a previously recognised hvKp strain with known virulence factors, and to rule out a novel emerging hypervirulent strain. However, there remain technical issues and limitations; the high-accuracy basecalling required for accurate genotyping takes substantial computing resources and clock time $(\geq 24$ hours), and best results were achieved with a model that had been previously custom-trained on K. pneumoniae [13]. Once a high-quality assembly was achieved, we were able to rapidly extract very informative genotyping using the web-based userfriendly data analysis tool, Pathogenwatch, and to compare the genome to that of previously sequenced isolates.

In summary, this case study demonstrates the benefits of a rapid sequencing method to identify and characterise an unusual, highly pathogenic hvKp strain. Sequencing is becoming easier to perform and less expensive, and bioinformatics analysis tools are simpler to use, heralding the possibility of using this technology in routine clinical microbiology laboratories to rapidly characterise clinically important isolates.

\section{Data Availability}

The sequencing data from this study are available from FigShare, doi: 10.6084/m9.figshare.11770908.

\section{Ethical Approval}

As a retrospective review of a single case, ethical approval was not sought.

\section{Consent}

As the case was fatal, the patient was not able to consent for publication. Family members were unable to be contacted via telephone or via reported address. As the patient was an undocumented migrant, it was considered likely that family members or next of kin would be unavailable to discuss proxy consent to participate. In this case, approval was obtained from the hospital's clinical director of critical care.

\section{Conflicts of Interest}

All authors have no conflicts of interest to declare.

\section{Authors' Contributions}

All authors read and approved the final manuscript. CKM and FAK contributed equally to this work. KEH and MET contributed equally to this work. CKM, BC, and $\mathrm{PH}$ were involved in patient management. $\mathrm{RB}$ provided laboratory support. FAK, BW, RW, KH, and MET contributed to sequencing and data analysis.

\section{Acknowledgments}

The authors are grateful to the clinical and laboratory staff at Medway Maritime Hospital for assisting with the clinical care and laboratory investigation of the patient. MET was supported by the Academy of Medical Sciences, the Health Foundation, and the National Institute of Health Research Cambridge Biomedical Research Unit. KEH was supported by a Senior Medical Research Fellowship from the Sylvia and Charles Viertel Foundation of Australia. BW received funding from the National Institute for Health Research Cambridge Biomedical Research Centre at the Cambridge University Hospitals NHS Foundation Trust. The views expressed are those of the authors and not necessarily those of the NHS, the NIHR, or the Department of Health and Social Care.

\section{Supplementary Materials}

The Supplementary Materials file includes the following information: Sequencing methods and bioinformatic analysis. Table S1: Klebsiella pneumoniae isolates included in the phylogenetic tree. Table S2: Kleborate genotyping output for nanopore sequenced K. pneumoniae isolate. Supplementary references. (Supplementary Materials)

\section{References}

[1] T. A. Russo and C. M. Marr, "Hypervirulent Klebsiella pneumoniae," Clinical Microbiology Reviews, vol. 32, no. 3, 2019.

[2] Y.-T. Lin, L. K. Siu, J.-C. Lin et al., "Seroepidemiology of Klebsiella pneumoniae colonizing the intestinal tract of healthy Chinese and overseas Chinese adults in Asian countries," BMC Microbiology, vol. 12, no. 1, p. 13, 2012.

[3] S. Rodriguez-Villar, A. Fife, C. Baldwin et al., "Antibioticresistant hypervirulent Klebsiella pneumoniae causing community-acquired liver abscess: an emerging disease," Oxford Medical Case Reports, vol. 2019, no. 5, pp. 206-211, 2019.

[4] K. J. Roulston, T. Bharucha, J. F. Turton et al., "A case of NDM-carbapenemase-producing hypervirulent Klebsiella pneumoniae sequence type 23 from the UK," JMM Case Reports, vol. 5, no. 9, pp. 1-3, 2018.

[5] J. Van Keer, K. Van Keer, J. Van Calster, and I. Derdelinckx, "More than meets the eye: Klebsiella pneumoniae invasive liver abscess syndrome presenting with endophthalmitis," The Journal of Emergency Medicine, vol. 52, no. 6, pp. e221-e223, 2017.

[6] L. Surgers, A. Boyd, P.-M. Girard, G. Arlet, and D. Decré, "ESBL-producing strain of HypervirulentKlebsiella pneumoniaeK2, France," Emerging Infectious Diseases, vol. 22, no. 9, pp. 1687-1688, 2016.

[7] M. Kolmogorov, J. Yuan, Y. Lin, and P. A. Pevzner, "Assembly of long, error-prone reads using repeat graphs," Nature Biotechnology, vol. 37, no. 5, pp. 540-546, 2019.

[8] R. Vaser, I. Sović, N. Nagarajan, and M. Šikić, "Fast and accurate de novo genome assembly from long uncorrected reads," Genome Research, vol. 27, no. 5, pp. 737-746, 2017.

[9] M. M. C. Lam, K. L. Wyres, L. M. Judd et al., "Tracking key virulence loci encoding aerobactin and salmochelin siderophore synthesis in Klebsiella pneumoniae," Genome Medicine, vol. 10, no. 1, 2018.

[10] B. D. Ondov, T. J. Treangen, P. Melsted et al., "Mash: fast genome and metagenome distance estimation using MinHash," Genome Biology, vol. 17, no. 1, 2016.

[11] M. M. C. Lam, K. L. Wyres, S. Duchêne et al., "Population genomics of hypervirulent Klebsiella pneumoniae clonal- 
group 23 reveals early emergence and rapid global dissemination," Nature Communication, vol. 9, no. 1, 2018.

[12] K.-M. Wu, L.-H. Li, J.-J. Yan et al., "Genome sequencing and comparative analysis of Klebsiella pneumoniae NTUH-K2044, a strain causing liver abscess and meningitis," Journal of Bacteriology, vol. 191, no. 14, pp. 4492-4501, 2009.

[13] R. R. Wick, L. M. Judd, and K. E. Holt, "Performance of neural network basecalling tools for Oxford nanopore sequencing," Genome Biology, vol. 20, no. 1, 2019.

[14] M. M. C. Lam, R. R. Wick, K. L. Wyres et al., "Genetic diversity, mobilisation and spread of the yersiniabactinencoding mobile element ICEKp in klebsiella pneumoniae populations," Microbial Genomics, vol. 4, no. 9, 2018.

[15] C. M. Marr and T. A. Russo, "Hypervirulent Klebsiella pneumoniae: a new public health threat," Expert Review of Anti-Infective Therapy, vol. 17, no. 2, pp. 71-73, 2019.

[16] L. K. Siu, K.-M. Yeh, J.-C. Lin, C.-P. Fung, and F.-Y. Chang, "Klebsiella pneumoniae liver abscess: a new invasive syndrome," The Lancet Infectious Diseases, vol. 12, no. 11, pp. 881-887, 2012.

[17] I. R. Lee, J. S. Molton, K. L. Wyres et al., "Differential host susceptibility and bacterial virulence factors driving Klebsiella liver abscess in an ethnically diverse population," Scientific Reports, vol. 6, 2016.

[18] C.-T. Fang, S.-Y. Lai, W.-C. Yi, P.-R. Hsueh, K.-L. Liu, and S.-C. Chang, "Klebsiella pneumoniae genotype K1: an emerging pathogen that causes septic ocular or central nervous system complications from pyogenic liver abscess," Clinical Infectious Diseases, vol. 45, no. 3, pp. 284-293, 2007. 